$16^{\text {th }}$ International Congress of Metrology, 08003 (2013)

DOI: $10.1051 /$ metrology/201308003

(C) Owned by the authors, published by EDP Sciences, 2013

\title{
Effect of form errors in datum features on evaluated geometries
}

\author{
Alistair Forbes ${ }^{1, a}$, Alan Wilson ${ }^{1}$, Per Saunders $^{2}$, and Nick Orchard ${ }^{2}$ \\ 1 National Physical Laboratory, Hampton Road, Teddington, Middlesex, TW11 0LW, UK \\ 2 Rolls-Royce plc, Gipsy Patch Lane, Filton, Patchway, Bristol BS34 7QE, UK
}

\begin{abstract}
In coordinate metrology a common task is to align the frame of reference of the work piece, as represented by the coordinates gathered by a coordinate measuring system, with that associated with its specification. The positions of geometric features of a work piece are often specified relative to datum features that define an axis system or frame of reference for the work piece. For an ideal geometry and ideal measuring system, subsequent calculations will not depend on which datum features are used to define the frame of reference. For real geometries, the presence of form error associated with the datum surfaces means that different measurement strategies will lead to different definitions of the frames of reference and therefore different estimates of the positions of other geometric features associated with the work piece. In this paper we define approaches for evaluating the contribution of form error associated with datum features to the uncertainties associated with geometric features derived from coordinate data.
\end{abstract}

\section{Introduction}

Coordinate measuring machines $(\mathrm{CMMs})$ are one of the most important tools in form and tolerance assessment in precision engineering. They are used to estimate how close a manufactured work piece is to its ideal geometry, as specified by a technical drawing or CAD specification. In order to be able to relate the work piece to its design, it is necessary to align the frame of reference of the work piece, as represented by the coordinates gathered by the CMM, with that associated with its specification.

The position, i.e., location and orientation, of geometric features of a work piece are often specified relative to datum features that define an axis system or frame of reference for the work piece. For example, a work piece might be associated with three planar surfaces that are nominally flat and mutually orthogonal. Measuring three points on one plane, two points on another and one point on the remaining surface is sufficient to fix the six degrees of freedom associated the definition of the frame of reference. For an ideal geometry and ideal measuring system, subsequent calculations will not depend on which six measured points are used to define the frame of reference. For real geometries and measuring systems, the effect of form error and squareness errors, i.e., non-ideal geometry, associated with the datum surfaces means that different measurement strategies will lead to different definitions of the frame of reference and therefore different estimates of the positions of other geometric features associated with the work piece. In section 2 , we discuss approaches to evaluating the uncertainties associated with coordinate data gathered from engineering surfaces and in section 3 we show how these uncertainties can be propagated through to derived geometric fea-

\footnotetext{
a e-mail: alistair.forbes@npl.co.uk
}

tures. Section 4 discusses approaches for evaluating the contribution of form error associated with datum features to the uncertainties associated with geometric features and includes a numerical example related a multi-feature artefact designed by Rolls-Royce. Our summary and concluding remarks are given in section 5 .

\subsection{Notation}

Given point coordinates $\boldsymbol{x}_{i}=\left(x_{i}, y_{i}, z_{i}\right)^{\mathrm{T}}$, where $i \in$ $I=\{1, \ldots, m\}$, the symbol $\boldsymbol{x}_{I}$ denotes the $3 m \times 1$ vector of point coordinates:

$$
\boldsymbol{x}_{I}=\left(x_{1}, y_{1}, z_{1}, x_{2}, y_{2}, z_{2}, \ldots, x_{m}, y_{m}, z_{m}\right)^{\mathrm{T}} .
$$

\section{Uncertainty associated with surface coordinate data}

Suppose $\boldsymbol{u} \mapsto \boldsymbol{s}(\boldsymbol{u}, \boldsymbol{a})$ defines a parametric curve or surface. The parameters $\boldsymbol{u}$ determine the position of a point on the surface and the parameters $\boldsymbol{a}$ determine the shape and position of the surface. We now assume that a set of measured coordinates, $\boldsymbol{x}_{I}$ generated according to the model

$$
\boldsymbol{x}_{i}=\boldsymbol{s}_{i}^{*}+f_{i} \boldsymbol{n}_{i}+\boldsymbol{e}_{i}+\boldsymbol{\epsilon}_{i}, \quad i \in I=\{1, \ldots, m\}
$$

where $\boldsymbol{x}_{i}$ is the $3 \times 1$ vector storing the observed coordinate data for the $i$ th point, $\boldsymbol{s}_{i}^{*}$ is a point lying on the surface with ideal geometry, $f_{i} \boldsymbol{n}_{i}$ is the form error at the $i$ th point in the direction of $\boldsymbol{n}_{i}$, the unit normal to the ideal geometry, and $f_{i}$ is the signed size of the form error, $\boldsymbol{e}_{i}$ is the parametric (systematic) error of the coordinate measuring system at $\boldsymbol{x}_{i}$, and $\boldsymbol{\epsilon}_{i}$ represents the random effects. 
Given a statistical characterisation of the quantities $\boldsymbol{f}=\left(f_{1}, \ldots, f_{m}\right)^{\mathrm{T}}, \boldsymbol{e}_{I}$, and $\boldsymbol{\epsilon}_{I}$ in terms of means and variances, then the means and variances associated with the point coordinates $\boldsymbol{x}_{I}$ can be determined using the law of propagation of uncertainty [1-3]. In particular, if

$$
\boldsymbol{f}_{I} \sim \mathrm{N}\left(\mathbf{0}, V_{\boldsymbol{f}}\right), \quad \boldsymbol{e}_{I} \sim \mathrm{N}\left(\mathbf{0}, V_{\boldsymbol{e}}\right)
$$

and

$$
\boldsymbol{\epsilon}_{I} \sim \mathrm{N}\left(\mathbf{0}, \sigma_{M}^{2} I\right)
$$

then the variance matrix $V_{I}$ associated with $\boldsymbol{x}_{I}$ is given by

$$
V_{I}=N^{\mathrm{T}} V_{\boldsymbol{f}} N+V_{\boldsymbol{e}}+\sigma_{M}^{2} I
$$

where $N$ is the $m \times 3 m$ block diagonal matrix storing $\boldsymbol{n}_{i}^{\mathrm{T}}$ in the $1 \times 3$ diagonal blocks.

If the measuring system is a coordinate measuring machine (CMM), then the systematic error $\boldsymbol{e}_{i}$ is often modelled in terms of the kinematic (or parametric) errors arising from the non-ideal geometry relating to scale errors, straightness and rotational errors $[9,11]$. In this case, $\boldsymbol{e}_{i}=\boldsymbol{e}\left(\boldsymbol{x}_{i}, \boldsymbol{b}\right)$ where $\boldsymbol{e}(\boldsymbol{x}, \boldsymbol{b})$ describes the kinematic error model as a function of location in the CMM working volume involving parameters $\boldsymbol{b}=\left(b_{1}, \ldots, b_{p}\right)^{\mathrm{T}}$. For example, $\boldsymbol{b}$ could be the coefficients of polynomial functions describing the kinematic errors. If $\boldsymbol{b} \sim \mathrm{N}\left(\mathbf{0}, V_{\boldsymbol{b}}\right)$, then

$$
V_{\boldsymbol{e}}=E V_{\boldsymbol{b}} E^{\mathrm{T}}
$$

where $E$ is the $3 m \times p$ matrix of the partial derivatives of $\boldsymbol{e}\left(\boldsymbol{x}_{i}, \boldsymbol{b}\right)$ with respect to $\boldsymbol{b}$.

While it may be valid to model the form errors $\boldsymbol{f}$ as associated with a multi-normal distribution $\boldsymbol{f} \sim$ $\mathrm{N}\left(\mathbf{0}, \sigma_{F}^{2} I\right)$, it may be more realistic to impose some spatial correlation [7] on them to reflect the fact that the form error is likely to vary smoothly. For example, we can use a Gaussian processes model [10] that specifies the covariance $\operatorname{cov}\left(f, f^{\prime}\right)$ of form errors $f$ and $f^{\prime}$ at locations $s$ and $\boldsymbol{s}^{\prime}$ on the surface is given by

$$
\operatorname{cov}\left(f, f^{\prime}\right)=k\left(s, s^{\prime}\right)=\sigma_{F}^{2} \exp \left\{-\frac{1}{2 \lambda^{2}}\left\|s-s^{\prime}\right\|^{2}\right\}
$$

where $\lambda$ defines length scale over which the correlation operates. For this type of model, the $i j$ th element of $V_{\boldsymbol{f}}$ is given by $k\left(\boldsymbol{s}_{i}^{*}, \boldsymbol{s}_{j}^{*}\right)$. In practice, this latter expression is usually approximated by $k\left(\boldsymbol{x}_{i}, \boldsymbol{x}_{j}\right)$.

\section{Uncertainty model associated with geometric features}

Suppose, as before, that $\boldsymbol{u} \mapsto \boldsymbol{s}(\boldsymbol{u}, \boldsymbol{a})$ defines a parametric curve or surface (e.g. a circle or cylinder). The least squares best-fit surface to data $\boldsymbol{x}_{I}$ can be found by minimising

$$
\sum_{i \in I} d_{i}^{2}\left(\boldsymbol{x}_{i}, \boldsymbol{a}\right), \quad d_{i}\left(\boldsymbol{x}_{i}, \boldsymbol{a}\right)=\left(\boldsymbol{x}_{i}-\boldsymbol{s}\left(\boldsymbol{u}_{i}^{*}, \boldsymbol{a}\right)\right)^{\mathrm{T}} \boldsymbol{n}_{i},
$$

where $\boldsymbol{u}_{i}^{*}$ specifies the point $s_{i}^{*}=s^{*}\left(\boldsymbol{u}_{i}^{*}, \boldsymbol{a}\right)$ on the surface closest to $\boldsymbol{x}_{i}$ and $\boldsymbol{n}_{i}$ is the normal vector at $\boldsymbol{s}_{i}^{*}$.

Let $J$ be the Jacobian matrix at the solution $\boldsymbol{a}^{*}$, $J_{i j}=\partial d_{i} / \partial a_{j}$ and let

$$
J=Q R=\left[\begin{array}{ll}
Q_{1} & Q_{2}
\end{array}\right]\left[\begin{array}{c}
R_{1} \\
\mathbf{0}
\end{array}\right]
$$

be its QR factorisation $[6,8]$. Then

$$
S=R_{1}^{-1} Q_{1}^{\mathrm{T}} N
$$

is the sensitivity of the solution parameters $\boldsymbol{a}$ to the data $\boldsymbol{x}_{I}$ so that the variance matrix $V_{\boldsymbol{a}}$ associated with the fitted parameters is derived from $V_{I}$ according to

$$
\begin{aligned}
V_{\boldsymbol{a}} & =S V_{I} S^{\mathrm{T}}=S\left(N^{\mathrm{T}} V_{\boldsymbol{f}} N+V_{\boldsymbol{e}}+\sigma_{M}^{2} I\right) S^{\mathrm{T}}, \\
& =R_{1}^{-1} Q_{1}^{\mathrm{T}} V_{\boldsymbol{f}} Q_{1} R_{1}^{-\mathrm{T}}+S V_{\boldsymbol{e}} S^{\mathrm{T}}+\sigma_{M}^{2}\left(R_{1}^{\mathrm{T}} R\right)^{-1} .
\end{aligned}
$$

This latter equation decomposes of the variance matrix associated with the fitted parameters into its components arising from form error, and systematic effects and random effects associated with the measuring system.

Similarly, the uncertainties associated with the coordinate data can be propagated through to the vector of residual distances $\boldsymbol{d}$, where $d_{i}=d\left(\boldsymbol{x}_{i}, \boldsymbol{a}\right)$ evaluated at the solution, according to

$$
\begin{aligned}
V_{\boldsymbol{d}} & =D V_{I} D^{\mathrm{T}}, \\
& =Q_{2} Q_{2}^{\mathrm{T}} V_{\boldsymbol{f}} Q_{2} Q_{2}^{\mathrm{T}}+D V_{\boldsymbol{e}} D^{\mathrm{T}}+\sigma_{M}^{\mathrm{T}} Q_{2} Q_{2}^{\mathrm{T}}
\end{aligned}
$$

with $D=Q_{2} Q_{2}^{\mathrm{T}} N$, where $Q_{2}$ arises from the QR factorisation of $J$ in (2).

Inferences about the fitness of purpose of a manufactured artefact are often made on the basis of the evaluated parameters $\boldsymbol{a}$ and residual distances $\boldsymbol{d}$, but these are only estimated on the basis of measured data subject to systematic and random effects and it is important to quantify how well these estimates have been determined. The uncertainty calculations described above allow such decisions to take into account the contribution of the various influence factors. Figure 1 illustrates the relationship of the fitted ideal geometry to the true geometric surface.

\section{Evaluated geometries relative to datum features}

In measuring an artefact, it is often necessary to measure datum surfaces associated with the artefact and then use these measurements to provide the coordinate data in a frame of reference derived from the datum surfaces. For example, this alignment to datum features is usually necessary in order compare the artefact geometry with that specified in a design drawing or CAD specification.

The datum features are chosen to provide six constraints to fix the frame of reference. For example, the 


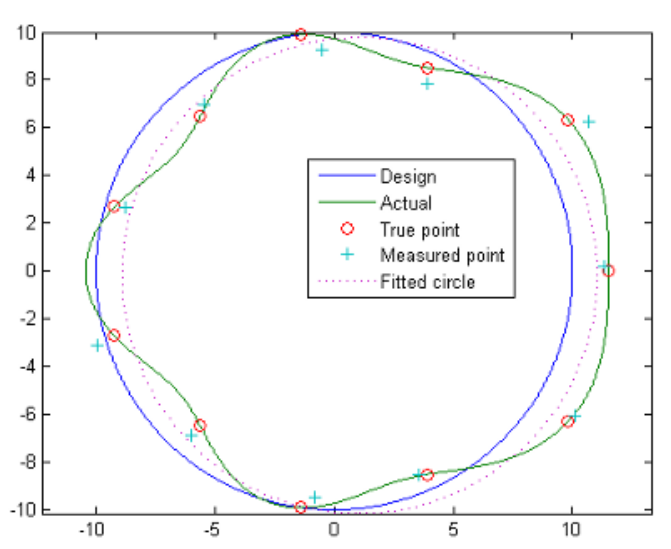

Fig. 1. Relationship between evaluated geometry and actual geometry taking into account the influence of form error and systematic and random effects associated with the measuring system

datum features may be three planes that, nominally, are mutually orthogonal. Measuring three points on one plane, two on a second and one on the third can be sufficient to assign the frame of reference. A plane is defined by three parameters, and threes plane are defined by a total of nine parameters. The measurement of six points only eliminates six degrees of freedom. The other three degrees of freedom are eliminated if we assume that the planes are mutually orthogonal. If the planes have form error, then choosing a different set of six points will generally define a slightly different frame of reference. The effect of systematic and random effects associated with the measurement system will also have influence in the defined frame of reference. In this section, we provide a general approach to evaluating the influence of these effects on the defined frame of reference and on subsequent calculations.

In order to describe the concepts, we use a multifeature artefact designed by Rolls-Royce and pictured in figure 2. The artefact has a number of features including a planar upper surface (three parameters), a line feature on one side (two parameters), a central circular spiggot (three parameters), a scallop, i.e., an arc of a circle on one side (three parameters), a conical feature centred around the central spiggot (six parameters) and six circular holes (three parameters each). Some of these features have been manufactured to include specified form errors. To the $k$ th feature, we associate the $k$ th set of feature parameters $\boldsymbol{a}_{k}$, and assemble all the feature parameters into a single vector a.

The preferred frame of reference of the complete geometry is specified by six constraints of the form

$$
\boldsymbol{c}(\boldsymbol{a})=\boldsymbol{c}_{0}
$$

For example, we can constrain the normal of a plane to be parallel to the $z$-axis and the plane to pass through the origin; this imposes three constraints.

Suppose that coordinate data $\boldsymbol{x}_{I}$ is measured by a coordinate measuring system and that the coordinates are given in an arbitrary frame of reference. We assume that the measured points are sufficient to deter-
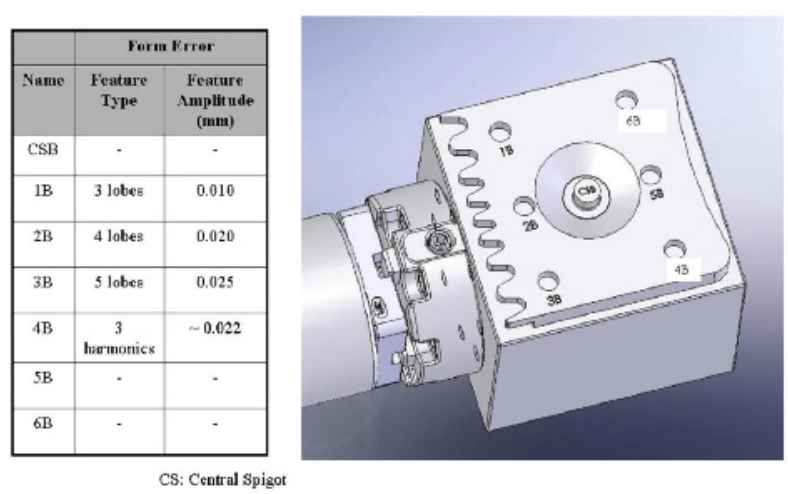

Fig. 2. Rolls-Royce multi-feature artefact.

mine estimates of the parameters $\boldsymbol{a}_{k}$ associated with the datum features. In order to conform to the frame of reference constraint (3), we seek a rigid body transformation

$$
\hat{\boldsymbol{x}}_{i}=T\left(\boldsymbol{x}_{i}, \boldsymbol{t}\right),
$$

such that the fitted feature estimates $\hat{\boldsymbol{a}}_{k}=\hat{\boldsymbol{a}}_{k}\left(\boldsymbol{x}_{I}, \boldsymbol{t}\right)$ derived from the transformed data set $\hat{\boldsymbol{x}}_{I}$ conforms to (3), i.e.,

$$
\boldsymbol{c}\left(\hat{\boldsymbol{a}}\left(\boldsymbol{x}_{I}, \boldsymbol{t}\right)\right)=\boldsymbol{c}_{0} .
$$

The equation (4) implicitly defines the transformation parameters $\boldsymbol{t}=\boldsymbol{t}\left(\boldsymbol{x}_{I}\right)$ as functions of $\boldsymbol{x}_{I}$ and the uncertainty associated with $\boldsymbol{x}_{I}$ can be propagated through to $\boldsymbol{t}$. The transformed data set $\hat{\boldsymbol{x}}_{I}=T\left(\boldsymbol{x}_{I}, \boldsymbol{t}\left(\boldsymbol{x}_{I}\right)\right)$ is then used to evaluate the parameters $\hat{\boldsymbol{a}}$ associated with all the geometric features. The transformed set is a function of $\boldsymbol{x}_{I}$, so uncertainty propagates through to $\hat{\boldsymbol{x}}_{I}$, and hence to $\hat{\boldsymbol{a}}$, with evaluated variance matrices $\hat{V}_{I}$, and $\widehat{V}_{\boldsymbol{a}}$, respectively. Note that if $X$ is the $3 m \times 3 m$ sensitivity matrix of $\hat{\boldsymbol{x}}_{I}$ with respect to $\boldsymbol{x}_{I}$, then

$$
\widehat{V}_{I}=X V_{I} X^{\mathrm{T}}
$$

Furthermore, $X$ has the form

$$
X=G+K H
$$

where $G$ is the $3 m \times 3 m$ block diagonal matrix whose $i$ th $3 \times 3$ block stores

$$
\frac{\partial T}{\partial \boldsymbol{x}}\left(\boldsymbol{x}_{i}, \boldsymbol{t}\right), \quad i \in I,
$$

$K$ is the $3 m \times 6$ matrix storing

$$
\frac{\partial T}{\partial \boldsymbol{t}}\left(\boldsymbol{x}_{i}, \boldsymbol{t}\right), \quad i \in I
$$

and $H$ is the $6 \times 3 m$ matrix storing

$$
\frac{\partial \boldsymbol{t}}{\partial \boldsymbol{x}_{i}}, \quad i \in I
$$

$I=\{1, \ldots, m\}$. Thus, calculations with $X$ can be performed compactly and efficiently in terms of the diagonal blocks of $G$ and the compact matrices $K$ and $H$. 
Table 1. Uncertainties associated with datum features i) three plane parameters, ii) two line parameter and iii) three circle parameters. The second and forth columns relate to variance matrix $V_{I}$ in the CMM frame of reference for low and moderate form error, while the third and fifth columns correspond to $\widehat{V}_{I}$ for the datum frame of reference.

\begin{tabular}{|c||r|r|r|r|}
\hline$z /(0.001 \mathrm{~mm})$ & 0.51 & 0 & 2.29 & 0 \\
$\alpha /(0.001 \mathrm{rad})$ & 0.02 & 0 & 0.07 & 0 \\
$\beta /(0.001 \mathrm{rad})$ & 0.01 & 0 & 0.06 & 0 \\
\hline$y /(0.001 \mathrm{~mm})$ & 0.99 & 1.11 & 0.99 & 7.15 \\
$\gamma /(0.001 \mathrm{rad})$ & 0.01 & 0 & 0.01 & 0 \\
\hline$x_{0} /(0.001 \mathrm{~mm})$ & 0.50 & 0 & 7.08 & 0 \\
$y_{0} /(0.001 \mathrm{~mm})$ & 0.50 & 0 & 7.08 & 0 \\
$r_{0} /(0.001 \mathrm{~mm})$ & 0.36 & 0.36 & 5.01 & 5.01 \\
\hline
\end{tabular}

\subsection{Numerical example based on the Rolls-Royce multi-feature artefact}

There a number of ways of using the features of the multi-feature artefact, figure 2, to specify a frame of reference. The calculations below relate to the following choices i) the upper datum surface coincides with the plane $z=0$, constraining translation along the $z$ axis and rotations about the $x$ - and $y$-axis, ii) the datum side line is parallel to the $x$-axis, constraining rotation about $z$, and iii) the spiggot centre coincides with $x_{0}=y_{0}=0$, constraining translations along the $x$ - and $y$-axis. The simulated measurement strategy involves gathering 5 points on upper datum face, 3 points on datum side line, 4 points on spiggot, 4 points for each of six the holes, 5 points on the scallop feature and 8 points, 4 on each of two circles on the cone. The associated features are plane, line, circle, and cone. The influence factors modelled are CMM scale and squareness errors, $[4,5]$, CMM random effects, and form error for each feature. The calculations derive the uncertainties in the feature parameters calculated according to the frame of reference of the CMM and that imposed by the frame of reference constraints associated with the datum features. The calculations are based on low and moderate form error associated with the datum features.

Uncertainties associated with datum features i) three plane parameters, ii) two line parameters and iii) three circle parameters are shown in table 1 . The second and fourth columns relate to variance matrix $V_{I}$ in the CMM frame of reference for low and moderate form error, while the third and fifths columns correspond to $\widehat{V}_{I}$ for the datum frame of reference. In the datum frame of reference, 6 of the parameters are fixed and have zero uncertainty. The constraints have the effect of redistributing the uncertainties amongst those parameters that are unconstrained. Table 2 provides the uncertainties associated with the hole features while table 3 gives the uncertainties associated the scallop and cone features. The uncertainties associated with the $x$-coordinate and radius of the scallop feature are large due to the fact the only a relatively small arc of the circle is measured.
Table 2. Uncertainties associated with centre coordinates of the six hole features. The units are micrometres. The first and third columns relate to variance matrix $V_{I}$ in the CMM frame of reference for low and moderate form error, while the second and fourth columns correspond to $\widehat{V}_{I}$ for the datum frame of reference.

\begin{tabular}{|l|l|l|r|}
\hline 3.65 & 3.71 & 3.65 & 7.98 \\
3.61 & 3.66 & 3.61 & 7.96 \\
2.51 & 2.51 & 2.51 & 2.51 \\
\hline 7.10 & 7.12 & 7.10 & 10.02 \\
7.08 & 7.11 & 7.08 & 10.02 \\
5.01 & 5.01 & 5.01 & 5.01 \\
\hline 8.53 & 8.56 & 8.53 & 11.10 \\
8.52 & 8.54 & 8.52 & 11.08 \\
6.01 & 6.01 & 6.01 & 6.01 \\
\hline
\end{tabular}

Table 3. Uncertainties associated with the scallop (first three rows) and cone features (remaining rows). The parameters in rows $1-5$ and 9 correspond to lengths and are in units of micrometres. The other parameters are in units of 0.001 radians. The first and third columns relate to variance matrix $V_{I}$ in the CMM frame of reference for low and moderate form error, while the second and fourth columns correspond to $\widehat{V}_{I}$ for the datum frame of reference.

\begin{tabular}{|r|r|r|r|}
\hline 43.11 & 43.12 & 43.11 & 43.69 \\
3.95 & 4.90 & 3.95 & 8.60 \\
42.79 & 42.79 & 42.79 & 42.79 \\
\hline 1.80 & 1.87 & 1.80 & 7.31 \\
1.80 & 1.87 & 1.80 & 7.31 \\
0.09 & 0.09 & 0.09 & 0.11 \\
0.09 & 0.09 & 0.09 & 0.11 \\
0.06 & 0.06 & 0.06 & 0.06 \\
1.15 & 1.45 & 1.15 & 4.14 \\
\hline
\end{tabular}

\section{Summary and concluding remarks}

In this paper we have discussed the propagation of uncertainties associated with form error, CMM systematic and random effects through to feature parameters and residual distances. The approach is quite generic and requires only the evaluation of the sensitivity matrices. If necessary, numerical techniques such a finite differences can be employed in their evaluation. We have also considered effect of form errors on datum features on evaluated geometries described in a frame of reference derived from the datum features. The approach enables their uncertainty contributions to be evaluated so that i) tolerances on datum features can be informed by their likely effect on evaluated geometries, and ii) different datum-ing strategies can be compared.

\section{Acknowledgements}

This work was supported by the UK's National Measurement Systems programmes for Engineering Measurement and Mathematics and Modelling. 


\section{References}

1. BIPM, IEC, IFCC, ILAC, ISO, IUPAC, IUPAP, and OIML. Evaluation of measurement data Guide to the expression of uncertainty in measurement. Joint Committee for Guides in Metrology, JCGM 100:2008.

2. BIPM, IEC, IFCC, ILAC, ISO, IUPAC, IUPAP, and OIML. Evaluation of measurement data Supplement 2 to the "Guide to the expression of uncertainty in measurement" - extension to any number of output quantities. Joint Committee for Guides in Metrology, JCGM 101:2011.

3. M. G. Cox and P. M. Harris. SSfM Best Practice Guide No. 6, Uncertainty evaluation. Technical Report MS 6, National Physical Laboratory, Teddington, UK, 2010.

4. A. B. Forbes. Surface fitting taking into account uncertainty structure in coordinate data. Measurement Science and Technology, 17:553-558, 2006.

5. A. B. Forbes. Uncertainty evaluation associated with fitting geometric surfaces to coordinate data. Metrologia, 43(4):S282-S290, August 2006.

6. A. B. Forbes. Parameter estimation based on least squares methods. In F. Pavese and A. B. Forbes, editors, Data modeling for metrology and testing in measurement science, pages 147-176, New York, 2009. Birkhäuser-Boston.

7. A. B. Forbes. Uncertainty associated with form assessment in coordinate metrology. Int. J. of Metrol. and Qual. Eng., 4:17-22, 2013.

8. G. H. Golub and C. F. Van Loan. Matrix Computations. John Hopkins University Press, Baltimore, third edition, 1996.

9. H. Kunzmann, E. Trapet, and F. Waldele. A uniform concept for calibration, acceptance test and periodic inspection of co-ordinate measuring machines using reference objects. Annals of the CIRP, 39:561-564, 1990.

10. C. E. Rasmussen and C. K. I. Williams. Gaussian Processes for Machine Learning. MIT Press, Cambridge, Mass., 2006.

11. G. Zhang, R. Ouyang, B. Lu, R. Hocken, R. Veale, and A. Donmez. A displacement method for machine geometry calibration. Annals of the CIRP, 37:515-518, 1988. 